\title{
The measurement of pupillary dilation: A facilitating apparatus
}

\author{
RICHARD HENKE \\ University of San Francisco, San Francisco, California 94117 \\ and \\ TAMAR GLOBERSON \\ Tel-Aviv University, Ramat-Aviv, Tel-Aviv, Israel
}

\begin{abstract}
The measurement of pupillary dilation has been facilitated by the invention of new sophisticated devices such as remote eye trackers. However, they are expensive and immobile, making their use impossible in studies with low budgets or those conducted outside of the laboratory. As a result, the conventional and tedious method of manually recording the pupil sizes from negatives of movie films, frame by frame, must still be used. The conventional method suffers from various limitations and is quite slow. The present article describes an optical apparatus that overcomes most of the limitations of the conventional method and, hence, increases the measurement efficiency.
\end{abstract}

The conventional approach to pupillometry consists of photographing one of the subject's eyes with a movie camera and subsequently measuring the pupil diameters on each individual frame of the processed film (Hakeram, 1967; Hess, 1972; Kahneman, 1973). These measurements are accomplished by comparing the projected pupil image with an appropriately enlarged scale placed on the projection screen. Mostly because of the inaccuracy and tedium of the measurement process, many types of direct-reading pupillometric recorders have been developed. The more sophisticated of these are computer-linked remote eye trackers, which are expensive and immobile and therefore suitable only for laboratory experiments, not for field studies. Consequently, in studies that have low budgets or are conducted outside of the laboratory, the less sophisticated and more conventional method of pupillometry must still be used.

The conventional method has several limitations: (1) Each pupil image must be individually aligned with the fixed scale lines on the screen. (2) The alignment and reading procedures are often difficult because of the somewhat diffuse edges of the pupil image. This arbitrariness in the definition of the "edge" can lead to systematic errors that can vary from day to day and from rater to rater. (3) Since reading the pupil diameter consists of comparing a diffuse edge with a scale and directly interpreting the numerical value, it is quite possible to inadvertently introduce a bias into the readings. The rater's desire for fairness can, therefore, lead to a great deal of equivocation. These limitations reduce measurement efficiency. Experienced raters

The authors are indebted to Shlomo Globerson for his help in the construction of the apparatus. Tamar Globerson is now at the Tel-Aviv University, University Campus, Ramat-Aviv, Tel-Aviv, Israel. Requests for reprints should be sent to Richard Henke, Department of Physics, University of San Fransisco, San Francisco, California 94117.

measure only 300 to 600 pictures per hour (Eilam, Note 1).

\section{THE DISPLACING DEVICE}

To overcome the noted limitations, the following apparatus was developed by Henke for a study conducted by Globerson (1976). The basic idea was suggested by a type of microscope eyepiece especially designed to measure the diameters of small round features. In such eyepieces, the separation of two overlapping images of the feature is adjustable and can be read from a scale when the circular images have been displaced to the point of tangency.

The apparatus is schematically demonstrated in Figure 1. The film (F) with the pupil image $(E)$ is passed through a standard slide-projector platen $(H)$. The film is wound from and to spools that are not shown. The pupil image is illuminated by a projection lamp (B) through a condenser lens (C). The condenser lens is placed so that it images the filament of $B$ onto the projector lens (L). It must be somewhat larger in

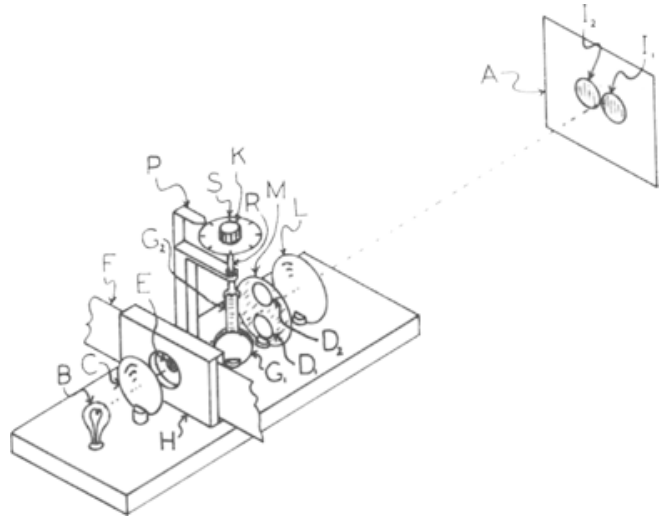

Figure 1. The apparatus: General view. 
diameter than the portion of the field that is to be projected on to the screen (A). With the portion of the apparatus described so far, we have the standard slideprojector configuration. In practice, $\mathrm{L}$ is not a singleelement lens as shown, but is actually a corrected multielement projector lens that is placed so that it focuses a clear image of the pupil onto the screen.

To convert the slide projector into a pupil diametermeasuring instrument, the following additional items are incorporated. Two flat pieces of optical glass $\left(G_{1}\right.$ and $\mathrm{G}_{2}$ ) are placed as close as possible to $L$ but in such a way that they can still be freely rotated, as shown in the figure. In addition, the mask (M) containing two diaphraghms $\left(D_{1}\right.$ and $\left.D_{2}\right)$ is inserted to isolate and make equal the portions of the beam going through $G_{1}$ and $G_{2}$, respectively.

The apparatus works as follows. The optical glasses, which intersect the beam at an angle, present horizontally displaced images of the pupil to L. Since they are rotated in opposite directions, the respective images of the pupil on the screen $\left(\mathrm{I}_{1}\right.$ and $\left.\mathrm{I}_{2}\right)$ are displaced in opposite directions. The upper optical glass $\left(\mathrm{G}_{2}\right)$ is attached to a rotating shaft (R) with an affixed scale (S). As the shaft is rotated by a knob (K), the amount of displacement of $G_{1}$ is adjusted until the two images $\left(I_{1}\right.$ and $I_{2}$ ) are just tangent. Then the angle of $G_{1}$ is indicated on $S$ by a pointer $(P)$; the displacement caused by $G_{1}$ can be computed from the rotation angle. When this displacement is added to the known and constant displacement produced by $G_{2}$, which remains fixed after the instrument is calibrated, the pupil diameter on the film is obtained.

The role of the two displacing glasses can be seen more clearly from the ray diagram shown in Figure 2. In the figure, only the central rays passing through $\mathrm{L}$ are shown to avoid confusion. The rays passing from the edges of the pupil image through $G_{1}$ and $G_{2}$ are shown, respectively, by solid and dashed lines. They then produce the displaced images $I_{1}$ and $I_{2}$.

In an actual device constructed from a standard slide projector, it will be found that when the projector lens is moved forward to allow the insertion of the displacing glasses and the diaphragms, the images $I_{1}$ and $\mathrm{I}_{2}$ are quite close to $\mathrm{L}$ and are fairly small. We compensated for this problem by using still another lens (not shown in the figures) to reimage the nearby, small pupil image into the final image on the screen. This second projector lens can be eliminated if $\mathrm{L}$ has a sufficiently long focal length to allow the images to be

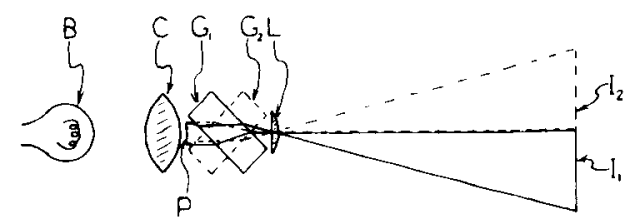

Figure 2. Optical aspects of the apparatus.

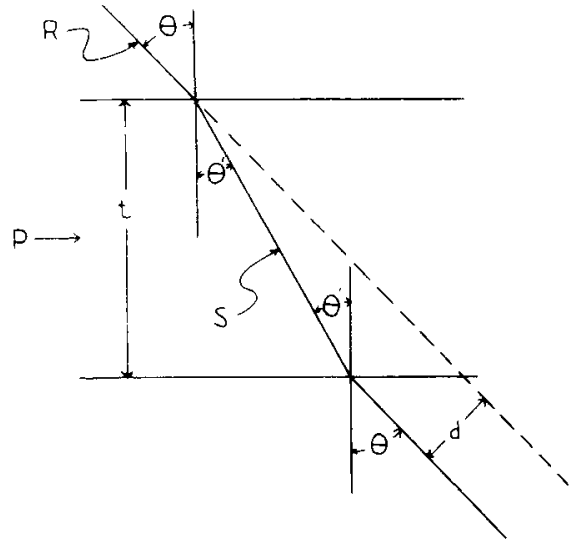

Figure 3. The displacement of a light ray by plane parallel refractive material.

focused on the screen with the additional lens-to-film distance required for the displacing device. The displacing device must be placed between the film and $\mathrm{L}$, however, to allow the measurements to be independent of the projector-to-screen distance.

An equation is derived in the following paragraphs that gives the quantitative value of the displacement (in millimeters, for example) as a function of angle of rotation of the glass $\left(G_{1}\right.$ or $\left.G_{2}\right)$. The apparatus is based on the optical principle of the displacement of a light ray by a plane parallel refractive material (Jenkins \& White, 1957, p. 20). This is demonstrated in Figure 3. Let $R$ be the light ray displaced by plane $P$, a flat optical glass with thickness $t$. The ray intersects the plane at an angle $\theta$ from the normal and is refracted through the plane at angle $\theta^{\prime}$. It obliquely travels a distance $s$ through the plane and is then refracted parallel to its original direction as it leaves the plane. As a result, the ray is laterally displaced the distance $d$.

The equation that transforms the angle measurement into the displacement measurement is derived using Snell's law of refraction (Jenkins \& White, 1957, p. 5). According to Snell's law, $\sin \theta / \sin \theta^{\prime}=n$, where $n$ is the index of refraction of the plane material (e.g., glass), and the index of the air is taken to be one. From the geometry in Figure $3, \mathrm{t}=\mathrm{s} \cos \theta^{\prime}$, and $\mathrm{d}=\mathrm{s} \sin \left(\theta-\theta^{\prime}\right)$. If ' $\theta$ ' and $s$ are eliminated from Snell's law and the two geometrical equations, the desired relationship between $\theta$ and $d$ is obtained:

$$
\mathrm{d}=\mathrm{t} \sin \theta\left(1-\frac{\cos \theta}{\sqrt{\mathrm{n}^{2}-\sin ^{2} \theta}}\right)
$$

\section{CALIBRATION OF THE DISPLACING APPARATUS}

The simplest approach to calibration is to individually measure or obtain the parameters in Equation 1. The thickness $t$ can be directly measured and, if $n$ is not available, a value of 1.53 should be sufficiently accurate for the typical crown optical glass. 
The angle $\theta$ must be measured from normal incidence on the optical glass. A convenient method of calibrating the angle scale is possible if the "fixed" optical glass is removable and can be rotated to zero the instrument. In this context, "fixed" refers to the fact that the fixed member is fixed during the measurement process but not during the calibration and zeroing of the instrument. With the fixed glass removed, the rotating glass is adjusted to make the two images on the screen exactly coincident. This is the normal incidence condition $(\theta=0)$, and the scale reading at this point, $\mathrm{SR}_{0}$, is the zero point for the angle measurement. Then, $\theta$ is given by:

$$
\theta=c\left(S R-S R_{0}\right)
$$

where SR is the scale reading and $c$ is the constant that converts from the units on the scale to the desired units for $\theta$ (usually degrees or possibly radians). The next step is to rotate the glass in the direction of decreasing SR until the moving image is displaced approximately $50 \%$ of the diameter of a typical pupil. (Each of the two glasses should provide approximately $50 \%$ of the total displacement in the final configuration.) For the instrument to achieve its maximum accuracy, this should occur when $\theta$ (Equation 2) is approximately in the range -20 through $-50 \mathrm{deg}$. If this is not possible, a glass of different thickness should be used. A thicker glass increases the displacement. The last step in the angle-calibration procedure is to replace the fixed glass (without moving the rotating one) and rotate it until the two images are again coincident. If the thickness of the two glasses are equal, this occurs when the two glasses are parallel. The current scale reading, $S R_{f}$, gives the value of the angle of the fixed glass, $\theta_{\mathrm{f}}=\mathrm{c}\left(\mathrm{SR}_{\mathrm{f}}-\mathrm{SR}_{0}\right)$, which will be constant and negative, since the fixed glass is rotated in the direction giving $S R$ less than $\mathbf{S R}_{0}$. The instrument is now properly zeroed and the fixed glass is left in place. When the rotating glass is rotated to an arbitrary angle $\theta$, the total relative displacement for the two optical glasses is the algebraic difference of their individual displacements,

$$
\begin{aligned}
d= & t \sin \theta\left(1-\frac{\cos \theta}{\sqrt{\mathrm{n}^{2}-\sin ^{2} \theta}}\right) \\
& -t \sin \theta_{\mathrm{f}}\left(1-\frac{\cos \theta_{\mathrm{f}}}{\sqrt{\mathrm{n}^{2}-\sin ^{2} \theta_{\mathrm{f}}}}\right)
\end{aligned}
$$

It should be noted that the two optical glasses need not be exactly the same thickness. In this case, the value of $t$ used in Equation 3 should be the thickness of the rotating glass.

Equation 3 gives the size of the pupil on the film. In practice, it is convenient to multiply by the ratio of the actual pupil size to the pupil size on the film. Then, Equation 3 gives the actual pupil size from the measured angle $\theta$. To obtain this objective, the best procedure is to photograph a scale at the location where the subject's eye will be for the pupil diameter-measurement exposures. Then, this calibration frame on the film is projected and the device used to "measure" the distance between the scale lines on the film image of the scale. The interval on the scale divided by the measured interval on the film (obtained from Equation 3) is the factor by which Equation 3 must be multiplied so that it will yield actual pupil size. In practice, this factor is calculated for several different intervals on the scale, and the average value is used.

In the present apparatus, a somewhat more complicated but more accurate approach was taken. The parameters in Equation 3 were obtained by making a series of measurements of a scale that was photographed in the same manner as were the experimental subjects in Globerson's (1976) study: A millimeter scale was pictured at the place where the subject's eye was pictured. In this way, the actual pupil size (in millimeters) could be inferred. The scale picture was displaced by the apparatus from .5 to $8.0 \mathrm{~mm}$, such that each millimetric displacement could be measured in its corresponding angle displacement. Each displacement was repeated three times, to enable computing an average, and was done on different pictures, to achieve a reliable calibration.

Table 1 demonstrates the calibration data. These measurements were plotted, and a curve of best fit (least squares) computed, resulting in the following transfer formula:

displacement $(\mathrm{mm})=$

$$
8.638 \sin \theta\left(1-\frac{\cos \theta}{\sqrt{2.241-\sin ^{2} \theta}}\right)+1.976
$$

where $\theta$ is given in terms of the scale reading (SR) by $\theta(\mathrm{deg})=1.8(\mathrm{SR}-19.12)$. Therefore, any reading $(\mathrm{SR})$

\begin{tabular}{|c|c|c|c|c|c|}
\hline \multirow{2}{*}{$\begin{array}{c}\text { Displace- } \\
\text { ment* }\end{array}$} & \multicolumn{4}{|c|}{ Scale Readings of Angles } & \multirow{2}{*}{$\begin{array}{c}\text { Computed } \\
\text { Displace- } \\
\text { ment* }\end{array}$} \\
\hline & 1 & 2 & 3 & Mean & \\
\hline .5 & 3.8 & 4.4 & 4.0 & 4.067 & .498 \\
\hline 1.0 & 8.9 & 8.8 & 9.1 & 8.933 & 1.021 \\
\hline 1.5 & 13.7 & 13.8 & 13.7 & 13.733 & 1.485 \\
\hline : & 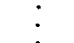 & 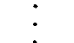 & 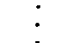 & & : \\
\hline 7.0 & $5 \dot{5.3}$ & 55.3 & 55.1 & 55.233 & 7.028 \\
\hline 7.5 & 57.1 & 57.5 & 57.2 & 57.267 & 7.524 \\
\hline 8.0 & 59.0 & 59.1 & 59.0 & 59.033 & 7.973 \\
\hline
\end{tabular}
from the apparatus could be translated into millimeters

Table 1 Calibration of the Displacing Device

*In millimeters. 
using Equation 4. A value of $S R=0$ gives a displacement of 0 . This results from the zeroing procedure in which the fixed glass is positioned to produce zero displacement when $S R=0$; i.e., $\theta_{\mathrm{f}}=-1.8 \times 19.12=-34.4$ deg. The value $n=1.497$ obtained is a quite reasonable one.

\section{ACCURACY OF THE APPARATUS}

The last column in Table 1 contains the value of displacement as computed from the mean scale reading. This value is to be compared with the actual displacement represented by the scale divisions given in the first column. The maximum difference between the readings is $.028 \mathrm{~mm}$ and the root-mean-square value for all of the differences is only $.021 \mathrm{~mm}$. This corresponds to only a few micrometers on the film and therefore demonstrates that the measurement accuracy is primarily limited by the resolution of the photography process.

\section{APPLICATION OF THE APPARATUS}

Two reliability measurements were made. (1) Betweenmeasurements reliability: During the measurement process, 251 pictures were measured five times each by the same rater. The coefficient of variation (SD/mean) was usually less than .01 and never exceeded .024 , even on the most ambiguous picture. That is, the relative error was usually less than $1 \%$ and never greater than $2.4 \%$. (2) Between-raters reliability: A total of 51 pictures were measured independently by two raters. The correlation coefficient between the two sets of measurements was $\mathrm{r}=.983$.

The reliability of the conventional method was computed to compare it with the reliability of the displacing apparatus. The two raters independently measured 49 pictures with a ruler (millimeters). The correlation coefficient between them was $r=.928$. This measure is also highly reliable, although perhaps less than the displacing device.

Validity of the new apparatus was established against the conventional one as follows: A set of 51 frames was measured twice, by the two methods (using a millimeter ruler in the conventional method). The correlation between the two sets of measures was $\mathrm{r}=.94$.

\section{ADVANTAGES}

The advantages of this instrument are as follows: (1) It is very easy to see when the images are tangent, especially if the picture is sharp. What is easily seen is that when the two images overlap, the overlapping area looks lighter (because more light falls on the same spot). When they are removed from each other, the area between them looks darker. When neither dark nor light areas are seen, the images are tangential. The
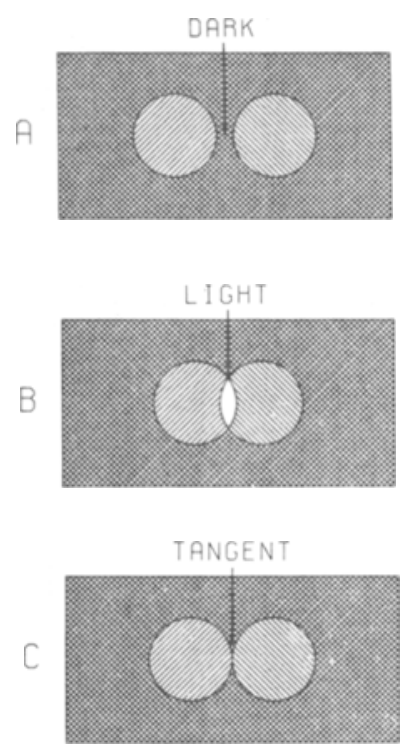

Figure 4. Three positions of a displaced pupil image: $A$ and $B$, incorrect positions; $C$, correct position.

three positions are demonstrated in Figure 4 . When the picture is not sharp, the advantage of the present method over the conventional one is most pronounced: It is easier to see the light and dark areas using the displacing device than to attempt to determine the tangent to a fuzzy borderline on a scale line, as is done in the conventional method. (2) The picture need not be enlarged a great deal. Any enlargement that is convenient to the eyes is sufficient for this method. Also, the measurement is always the same, at any projector-screen distance. (3) The projected image need not be adjusted so that it is tangent to a scale. It is positioned at any place on the screen and is measured there. (4) The method is easy on the eyes and on the rater's sitting position. The screen can be located at any distance, and the scale is located right beside the rater. (5) The process of measurement does not have to start from the zero point for each new frame. The pupil varies very little from frame to frame, hence the knob on the scale can be quickly adjusted to the new measurement. (6) The device is easy to construct at a low cost. (7) Because the measurements essentially consist of angles of the displacing device shaft, the measuring process could quite easily be semiautomated by attaching an encoder to the shaft. The angle values could then be transmitted to a recording device or computer at the touch of a button. In contrast, there is no convenient way of automating the conventional measurement procedure.

All of these factors enhance the convenience and efficiency of the measurement process. It is hard to estimate the rate of measurement because it depends on the quality of the photographs. However, in Globerson's (1976) study, even before practice, the rate 
was about 300 frames/h; with practice and with making motions more efficient, the rate was increased up to $1,000 \mathrm{frames} / \mathrm{h}$. This figure is a moderate overestimate, since it includes frames that were quickly scanned but not measured because they contained blinks or were out of focus. Such pictures are also considered in the estimations made on the conventional method, 300-600 frames/h for an experienced rater.

It seems clear that the displacing device is more convenient, efficient, and reliable than the conventional method.

\section{REFERENCE NOTE}

1. Eilam, Z. Personal communication, Department of Psychology, Tel-Aviv University, Israel, 1973.

\section{REFERENCES}

Globerson, T. Field independence, information processing capacity, and mental effort. Doctoral dissertation, University of California, Berkeley, 1976.

Ha Kerem, G. Pupillography. In P. H. Venables \& I. Martin (Eds.), Manual of psycho-physical methods. Amsterdam: North-Holland, 1967.

Hess, E. H. Pupillometrics. In N. S. Greenfield \& R. A. Sternbach (Eds.), Handbook of psychophysiology. New York: Holt, Rinehart, \& Winston, 1972.

Jenkins, F. A., \& White, H. E. Fundamentals of optics. New York: McGraw-Hill, 1957.

Kahneman, D. Attention and effort. Englewood Cliffs, N.J: Prentice Hall, 1973.

(Received for publication December 29, 1977; revision accepted December 29,1978 .) 